\title{
Could machine learning break the convection parameterization deadlock?
}

7 Corresponding author: Pierre Gentine (pg2328@columbia.edu)

- We use a global atmospheric model with embedded cloud resolving models (superparameterization) on an aquaplanet as a training dataset for a machine-learning algorithm that predicts the net effects of convective heating and moistening, as well as radiative transfer, including cloud-radiative feedbacks.

- The machine-learning algorithm can reproduce most of the key features of embedded

- The machine-learning algorithm is much more computationally efficient than a superparameterization, but exhibits reduced variance, especially in the lower troposphere. 


\section{Abstract}

Representing unresolved moist convection in coarse-scale climate models remains one of

21 the main bottlenecks of current climate simulations. Many of the biases present with

22 parameterized convection are strongly reduced when convection is explicitly resolved (i.e. in

23 cloud resolving models at high spatial resolution $\sim$ a kilometer or so). We here present a novel

24 approach to convective parameterization based on machine learning, using an aquaplanet with

25 prescribed sea surface temperatures as a proof of concept. A deep neural network is trained with

26 a superparameterized version of a climate model in which convection is resolved by thousands of

27 embedded 2D cloud resolving models. The machine learning representation of convection, which

28 we call the Cloud Brain (CBRAIN) can skillfully predict many of the convective heating,

29 moistening, and radiative features of superparameterization that are most important to climate

30 simulation, although an unintended side effect is to reduce some of the superparameterization

31 inherent variance. Since as few as three months' high frequency global training data prove

32 sufficient to provide this skill, the approach presented here opens up a new possibility for a

33 future class of convection parameterizations in climate models that are built "top-down", i.e. by

34 learning salient features of convection from unusually explicit simulations.

\section{Plain Language Summary}

The representation of cloud radiative effects and the atmospheric heating and moistening

37 due to moist convection remains a major challenge in current generation climate models, leading 38 to a large spread in climate prediction. Here we show that neural networks trained on a high39 resolution model in which moist convection is resolved can be an appealing technique to tackle 40 and better represent moist convection in coarse resolution climate models. 


\section{Introduction}

Convective parameterization remains one of the main roadblocks to weather and climate

43 prediction [Stevens and Bony, 2013; Medeiros et al., 2014; Sherwood et al., 2014; Bony et al.,

44 2015]. In fact, most of the inter-model spread in equilibrium climate sensitivity can be traced

45 back to the representation of clouds [Schneider et al., 2017]. Convective schemes exhibit

46 systematic biases in the vertical structure of heating and moistening, precipitation intensity, and

47 cloud cover [Daleu et al., 2015; 2016]. These errors, in turn, feed back onto larger-scale

48 circulations, deteriorating general circulation model (GCM) simulations and prediction skill

49 [Bony et al., 2015]. A challenge in current convective schemes is representing the transitions

50 between different types of convection, such as the transition from shallow to deep convection

51 [Khouider et al., 2003; Khouider and Majda, 2006; Khouider et al., 2010; Peters et al., 2013;

52 Couvreux et al., 2015; D'Andrea et al., 2014; Rochetin et al., 2014a; 2014b], which is especially

53 crucial to predicting both continental precipitation and modes of climate variability [Arnold et

$54 a l ., 2014]$. In addition, most convective parameterizations do not represent important processes,

55 such as convective aggregation, which are essential to accurately predicting the response of

56 clouds and precipitation to global warming, as well as modes of climate variability [Jeevanjee

57 and Romps, 2013; Wing and Emanuel, 2014; Arnold and Randall, 2015; Bony et al., 2015;

58 Bretherton and Khairoutdinov, 2015; Coppin and Bony, 2015; Muller and Bony, 2015].

59 A challenge in convective parameterization is the specification of the plume lateral 60 entrainment [Cohen, 2000; De Rooy et al., 2013; Sherwood and Hernández-Deckers, 2013; Yeo

61 and Romps, 2013; Tian and Kuang, 2016], its dependence on environmental conditions (e.g.,

62 free tropospheric dryness) [Derbyshire et al., 2004] and the role of subcloud layer organization

63 [D'Andrea et al., 2014; Naumann et al., 2017]. Entrainment is one of the major factors 
64 controlling climate sensitivity and explains, to a large extent, the intermodel spread in climate sensitivity in the tropics [Popke et al., 2013; Tomassini et al., 2014]. Entrainment also regulates some of the main features of tropical climate [Singh and $\left.O^{\prime} G o r m a n, 2013\right]$ such as the Inter

67 Tropical Convergence Zone (ITCZ) [Oueslati and Bellon, 2015], or modes of climate variability

68 [Bush et al., 2015] such as El Niño or the Madden Julian Oscillation (MJO) [Kim et al., 2012;

69 Feng et al., 2015]. In addition, the representation of the transition between shallow and deep convection is tightly related to changes in updraft entrainment [Del Genio and Wu, 2010;

71 D'Andrea et al., 2014], in part due to the organization of the subcloud layer by cold pools 72 [Khairoutdinov and Randall, 2006; D'Andrea et al., 2014]. The representation and understanding 73 of entrainment has defied a unified theory even though important progresses have been made in 74 recent years [Khouider et al., 2003; Khouider and Majda, 2006; De Rooy and Siebesma, 2010; 75 Khouider et al., 2010; Romps, 2010; Dawe and Austin, 2013; De Rooy et al., 2013; Peters et al., 76 2013; Sherwood and Hernández-Deckers, 2013; Yeo and Romps, 2013; Dorrestijn et al., 2015; 77 Romps, 2016].

78 Current generation climate models (and typical weather forecast models) with parameterized convection do not capture much of the degree of organization, nor do they represent mesoscale convective systems (MCS), [Hohenegger and Stevens, 2016] though the latter are likely essential

81 to accurate simulation and prediction of extreme rainfall events [Houze, 2004; Tan et al., 2015].

82 Finally, another challenge is that climate sensitivity is strongly related to the interaction between 83 deep and shallow convection [Bony et al., 2015], and the coupling between clouds, convection 84 and the large-scale circulation, which is currently poorly captured by parameterized convection 85 [Bony et al., 2015; Daleu et al., 2016; Hohenegger and Stevens, 2016; Nie et al., 2016]. 
Many of the previously mentioned problems related to the representation of convection are partly alleviated when using convective-permitting resolutions, i.e. at horizontal grid spacing of

$88 \sim 2 \mathrm{~km}$ or less. For instance, the transition between shallow and deep convection can be correctly 89 captured at convective permitting scale [Khairoutdinov and Randall, 2006; Khairoutdinov et al., 2009]. Convective aggregation is observed at convective permitting scale [Hohenegger and

91 Stevens, 2016] so that Cloud Resolving Models (CRMs) have been the tool of choice to 92 understand convective aggregation [Jeevanjee and Romps, 2013; Wing and Emanuel, 2014;

93 Arnold and Randall, 2015; Bony et al., 2015; Bretherton and Khairoutdinov, 2015; Coppin and

94 Bony, 2015; Muller and Bony, 2015]. CRMs (at convective permitting scales <2km) also 95 correctly reproduce MSCs and squall lines [Moncrieff and Liu, 2006; Taylor et al., 2009], as well 96 as extreme precipitation events driven by larger scale anomalies. Convective-permitting 97 simulations better represent modes of tropical climate variability [Arnold et al., 2014], shallow to 98 deep convection [Guichard et al., 2004] and mesoscale propagation [Hohenegger et al., 2015].

99 Therefore, modeling at convective-permitting scales is transformative to the representation of 100 convection. It is however impractical at present to use convective resolving resolution at global 101 scale for climate prediction given its computational requirements [Satoh et al., 2008]. While 102 Global Cloud Resolving Models (GCRMs) can be run easily for months, multidecadal 103 simulations are computationally challenging. To alleviate this problem, an interesting approach 104 has been to use cloud "superparameterization (SP)", which computes the subgrid vertical heating 105 and moistening profiles within a GCM grid cell by sampling a curtain of an embedded 2D CRM 106 that uses convective permitting resolution [Grabowski et al. 1999; Khairoutdinov et al., 2005]. 107 This has led to many successes such as the possibility to rectify the diurnal continental cycle, to 108 improve the representation of the $\mathrm{MJO}$, and to represent both some MCS propagation and some 
109 degree of aggregation, and reduce overly strong land-atmosphere coupling [Grabowski, 2001;

110 Khairoutdinov et al. 2005; Pritchard and Somerville, 2009; Benedict and Randall, 2009;

111 Pritchard et al. 2011; Randall, 2013; Kooperman et al., 2016a; 2016b; Sun and Pritchard, 2016;

112 Qin et al. 2018].

113 While promising, superparameterization is not without its own idealizations that also limit its

114 predictive ability and usefulness for climate simulation. For instance, restricting explicit

115 convection to two dimensions makes it difficult to represent momentum transport [Jung and

116 Arkawa, 2014; Arakawa 2011; Tulich 2015; Woelfle et al. 2018], and the limited CRM domain

117 extent artificially constrain vertical mixing efficiency [Pritchard et al. 2014]. Meanwhile, the

118 typical use of $1-4 \mathrm{~km}$ CRM horizontal resolution and 250-m vertical resolution cannot resolve

119 important boundary layer turbulence, lower tropospheric inversions, and associated entrainment

120 that are critical to low cloud dynamics [Parishani et al. 2017].

121 In light of this ongoing deadlock, we propose to use an alternative approach to convective 122 parameterization in which convection is represented using a machine-learning algorithm based 123 on Artificial Neural Networks (ANNs), trained on superparameterized simulations, called Cloud

124 Brain (CBRAIN). ANNs can approximate any non-linear deterministic function, a property 125 called the universal approximation theorem [Schmidhuber, 2015]. Clearly, parameterizing 126 convection appears as an ideal problem for the use of machine learning algorithms and especially 127 ANNs. Indeed, machine-learning algorithms have been used in many applications where a clear 128 physically-based algorithm could not be defined. Applications have included self-driving cars, 129 society games (chess and go) [Silver et al., 2016], speech recognition [Hinton et al., 2012], 130 object recognition and detection, medical detection of cancers [Khan et al., 2001; Zhou et al., $1312002 ;$ Karabatak and Ince, 2009], and genomics. There are also applications of ANNs to the 
132 geosciences, such as for rainfall prediction [Moazami et al., 2013; Miao et al., 2015; Tao et al.,

133 2016], soil moisture [Kolassa et al., 2013; 2016; 2017a; 2017b], and surface turbulent flux

134 retrievals [Jimenez et al., 2009; Jung et al., 2011; Alemohammad et a1., 2017]. Specifically, the

135 development of deep learning and Deep Neural Networks (DNN), i.e., those with multiple

136 hidden layers, has led to important developments in many different fields such as object

137 detection or game strategy learning [Dahl et al., 2011; Hinton et al., 2012; LeCun et al., 2015;

138 Silver et al., 2016; Tao et al., 2016]. One of the advantages of ANNs is that, once trained, they

139 are computationally efficient, as most of the computational burden is dedicated to the training 140 phase.

\section{Data}

SuperParameterized Community Atmosphere Model

To evaluate this idea, we use a well validated version of the SuperParameterized Community

144 Atmosphere Model (SPCAM3) in a simplified aquaplanet configuration with zonally symmetric

145 SSTs following a realistic meridional distribution [Andersen and Kuang, 2012]. The global

146 model uses a spectral dynamical core with approximately two-degree horizontal resolution (T42

147 triangular truncation) and 30 levels in the vertical. The CRM uses a simplified bulk one-moment

148 microphysics scheme and a Smagorinsky 1.5-order subgrid scale turbulence closure as described

149 by [Khairoudtinov et al., 2003] and shares the host GCM's vertical grid. For computational

150 efficiency and convenience we use the "micro-CRM" (8-column) CRM domain discussed by

151 Pritchard et al. (2014) for this proof of concept. Following a 3-month spinup period, we save

152 global data at the host global model timestep frequency (every 30 minutes) representing arterial

153 inputs to (and outputs from) each of 8,192 cloud-resolving arrays embedded SPCAM. The 154 simulation is run for two years, yielding around 140 million training samples per year. 


\section{Neural network setup}

156 We are using an ANN to predict SPCAM's total physics package tendencies, i.e. the

157 cumulative tendency produced by turbulence, convection and radiation. Rather than purely 158 isolating any of the above sub-tendencies from the CRM or GCM parameterizations, we chose a 159 holistic approach in representing their sum - that is, the arterial total heating and moistening 160 profiles that ultimately link a GCM's subgrid physics to its dynamical core. This has practical 161 advantages in that the individual physical sub-processes - turbulence, convection, microphysics, 162 and radiation - can interact in complex, non-linear ways. Approximating the net effect of such 163 interactions is one the big strengths of ANNs.

164 The ANN is written using the Python library Keras (https://keras.io), a high-level wrapper 165 around TensorFlow (http://www.tensorflow.org). The code for the ANN training as well as for 166 the validation and analysis below can be found at https://github.com/raspstephan/CBRAIN-

167 CAM. Training took on the order of 12 hours on a Graphical Processing Unit (GPU) (Nvidia 168 GTX 970). The first year of SP-CAM data was used for training, while the second year was used 169 for independent validation.

170 The feedforward ANNs consist of interconnected layers, each of which have a certain 171 number of nodes (Figure S 1). The input and output variables are listed in Table 1. The first layer 172 is the input layer, which in our case is a stacked vector containing the input variables including 173 their vertical variation for a specific column. The last layer is the output layer, which again is a 174 stacked vector of the four output vertical profile variables. All layers in between are called 175 hidden layers. Deep neural networks have more than one hidden layer. The activation function 176 the function acting on each node - is a weighted sum of the activations in all nodes of the 177 previous layer plus a bias term, passed through a non-linear activation function. Here, we used 
178 the Leaky Rectified Linear Unit (LeakyReLU) $a(x)=\max (0.3 x, x)$ as an activation function.

179 The output layer is purely linear without an activation function.

180 Training an ANN means optimizing the weight matrices and bias vectors that define it, to

181 minimize a loss function - in our case the mean squared error - between the ANN outputs and

182 the truth for a given input. The loss is computed for a shuffled (in space and time) mini-batch of

183 the training data with a batch size of 1024 samples. To reduce the loss, the gradient of the loss

184 function with respect to all weights and biases is computed using a backpropagation algorithm,

185 followed by a step down the gradient - i.e. stochastic gradient descent (SGD). In particular we 186 use a version of SGD called Adam [Kingma and Ba, 2014]. How much to step down the gradient

187 is determined by the learning rate. We started with a learning rate of $10^{-3}$, dividing it by 5 every 5

188 epochs (i.e. 5 passes through the entire training data set). In total we trained for 30 epochs.

189 For an ANN to train efficiently, all input values should be on the same order of magnitude.

190 For this purpose, for each input variable we subtracted the mean and divided by the standard 191 deviation, independently for each vertical level; not normalizing did not modify any results but 192 extended the duration of the training process. To make the outputs comparable we converted the 193 output variables (i.e. convective and radiative heating as well as convective moistening rates) to 194 common energy units.

\section{Results}

We start by testing how the amount of ANN parameters and their configuration impacts the 198 performance. Table S1 summarizes twelve separate ANN architectures tested. As a first metric 199 of skill we assess a mean squared error statistic computed across all four output variables, all 200 space, and all time during the second simulated year. That is, given knowledge of the inputs to 
201 each CRM, we measure the error across 143 million separate ANN predictions of the CRM

202 heating and moistening output profiles received by SPCAM's dynamical core, during a one-year

203 time period that was not included in the training dataset.

204 Figure S2a shows strong sensitivities to network architecture that underscore the importance

205 of the ANN design -- more parameters generally produce better scores and deeper networks give

206 better results, because they also allow for more non-linear interactions. For all subsequent

207 analyses we thus only use our best performing network -- a large, deep network with eight

208 hidden layers of 512 nodes each.

209 A key question for the generalizability of our approach is how much training data is needed.

210 To find out, we examine the effect of denying portions of the training data (Figure $\mathrm{S} 2 \mathrm{~b}$ ). As

211 expected, more training data does lead to better scores on the validation set. But, interestingly,

212 three months appear to be sufficient to yield most of the information (Figure S 2b). This suggests

213 promising potential to generalize our approach beyond an SPCAM demonstration testbed to

214 other simulation strategies that do even more justice to the true physics of moist convection.

215 Indeed, three-month simulations are practical even for global cloud resolving models or high-

216 resolution, 3D variants of SP. Due to the large amount of training data available to us we did not

217 see any serious signs of overfitting during the training samples and calibrations and training

218 statistics were very similar (not shown).

$219 \quad 4.2$ Evaluation of NN predictions

220 Latitude-longitude and pressure-latitude snapshots (Figure 1 and Figure 2) provide a good 221 qualitative starting point for evaluating the NN predictions (SUPPLEMENT VIDEOS). Overall, 222 the NN predictions agree remarkably well with the SP-CAM truth in terms of horizontal and 223 vertical structure. Lower tropospheric convective (turbulent and latent) heating and moistening 
224 associated with the intertropical convergence zone and extratropical cyclones occur at 225 approximately the correct geographic locations (Figure 1a-d). The radiative heating rates show 226 very good agreement, which is particularly impressive given the fact that there is no cloud 227 condensate information in the input, i.e. cloud-radiative feedback is all internal to the ANN. For 228 instance, ANN skillfully predicts the geographic location of shortwave absorption by water 229 vapor and regional cloud anomalies (Figure $1 \mathrm{~g}-\mathrm{h}$ ) as well as the vertical location of longwave 230 cooling maxima at the tops of subtropical boundary layer clouds and deep tropical clouds (Figure $2312 \mathrm{e}-\mathrm{f})$. However, one issue for the convective heating and particularly moistening rates is that the 232 NN predictions are smoother and do not exhibit as much of the variability as SP-CAM (internal 233 stochastic variability). Indeed, the ANN is by definition deterministic and thus cannot reproduce 234 any stochasticity.

235 To assess the quality of the predictions in more detail, we analyze $\mathrm{R}^{2}$, as well as error and 236 variance averaged over both time and horizontal dimensions to yield statistics for each level and 237 predicted variable (Figure 3). The radiative heating rates are well represented throughout the 238 column, particularly for shortwave heating. The convective tendencies interestingly show a 239 distinct profile with less predictive skill in the boundary layer and the stratosphere. In the 240 stratosphere, this lower skill is simply due to the near absence of convection at upper levels and 241 likely not a concern. In the boundary layer, the reasons for reduced skill are discussed more 242 below.

243 First, for a closer analysis of the skill in the troposphere we also look at spatial statistics. 244 Pressure-latitude maps of $\mathrm{R}^{2}$ and the standard deviation (Figure 4) reveal patches of especially 245 high skill in the mid-levels at the equator and mid-latitudes, which correspond to the locations of 246 the Inter Tropical Convergence Zone and the mid-latitude storm tracks. Since these are the 
247 locations of latent heating most fundamental to forcing the free tropospheric general circulation,

248 this is reassuring regarding the potential of CBRAIN to reproduce important heating and

249 moistening tendencies in future tests that could allow it to feedback with a dynamical core.

250 The skill in the boundary layer is significantly lower, again. One possibility is that this

251 reflects the difficulty in representing mesoscale effects and subcloud layer organization as well

252 as its memory [Mapes and Neale, 2011; D'Andrea et al., 2014]. SPCAM does include some

253 degree of convective aggregation [Arnold et al., 2015] and also carries memory of CRM

254 organization from one-time step to the next through the embedded CRM [Pritchard et al., 2011].

255 Our ANN does not include memory, as our objective was to mimic most current practice in

256 convective parameterization, which is local in space and time. Future versions could include

257 additional memory in the boundary layer, which would be worth exploring, although it requires

258 more computational expense. Another source of lower $\mathrm{R}^{2}$ is related to the higher internal

259 variability in SPCAM simulations compared to the ANN prediction, evident in Figure 1 and

260 Figure 2. This may be less of an issue in configurations that use larger, or 3D CRMs; the small-

261 extent 2D CRMs used here are known to throttle deep updrafts and lead to unrealistically intense

262 extremes [Pritchard et al., 2014]. But SPCAM has also been shown to being able to represent

263 some degree of stochasticity [Subramanian and Palmer, 2017], which, by definition, a

264 deterministic ANN cannot reproduce, so this issue may benefit from additional approaches. The

265 boundary layer and shallow convection tendencies, particularly for the moistening rate, are much

266 noisier and thus appear much more stochastic than at higher levels. In these lower levels, the

267 predictions here have significantly less variability in terms of its mean squared error low

268 function, which encourages the ANN to predict just an average value in cases where it is not

269 certain. 


\section{Discussion and conclusion}

271 We have demonstrated that machine learning, and neural networks in particular, can

272 skillfully represent many of the effects of unresolved clouds and convection, including their

273 vertical transport of heat and moisture and the interaction of radiation with clouds and water

274 vapor. The concept was proven in an idealized testbed using SPCAM over an aquaplanet. The

275 implication of the success in this context is that an approach like CBRAIN could glean the 276 advantages of GCRMs or high-resolution, 3D super-parameterizations not yet practical for 277 multidecadal climate simulations.

278 There are, however, important steps required for full implementation of CBRAIN in a GCM.

279 First, neural networks do not intrinsically preserve energy and moisture. This can be fine for 280 implementation in a weather forecast model but energy and moisture conservation are required

281 for climate prediction. Second, neural networks are inherently deterministic. It was shown here 282 that the resulting CBRAIN representation of heating and moistening tendencies was too smooth 283 compared to the original SPCAM field used for training, which is more variable especially in the 284 lower levels of the atmosphere (below 700hPA). An important next test to examine how 285 CBRAIN feeds back with the GCM's resolved scale dynamics and surface fluxes. A final 286 challenge is related to the fact that inherently a machine-learning algorithm is trained on existing 287 data. For climate prediction, the algorithm should be able to generalize to situations that have 288 potentially not been seen such as changes in trace gas profile and concentrations or aerosols, as 289 well as to continents, etc.

290 Notwithstanding the above challenges, we believe that our preliminary results motivate the 291 case that machine learning represents a powerful alternative to GCRMs or embedded-2D CRM 292 parameterizations. It is computationally efficient, even for relatively large networks. For 
293 instance, without specific optimization a preliminary test showed that CBRAIN was 10 times

294 faster than the "micro-CRM" form of SP used in our study. It would thus be several orders of

295 magnitude faster than an SP equipped with large, 3D, high-resolution domains, or a GCRM.

296 CBRAIN is also naturally fitted for data assimilation since computation of the adjoint is

297 straightforward and analytical, making it a natural candidate for operational weather forecasting.

298 CBRAIN could represent a useful alternative to current parameterizations, which have followed

299 a "bottom-up" deterministic strategy that still exhibits too many biases for satisfying prediction

300 of the future hydrological cycle. A "top-down" strategy that instead learns the realistic

301 complexity of simulated convection, as captured in short multi-month simulations at convection

302 permitting resolution, is an attractive alternative. As global temperature sensitivity to $\mathrm{CO}_{2}$ is

303 strongly linked to convective representation, this might also improve our estimates of future

304 temperature.

\section{Acknowledgments, Samples, and Data}

306 The neural network and analysis code can be found at https://github.com/raspstephan/CBRAIN-

307 CAM. The exact version of the code base used for this study is tagged grl_submission. The raw

308 SP-CAM output is very large (several TB) and available upon request to Prof. Mike Pritchard.

309 MP acknowledges funding from the DOE SciDac and Early Career Programs (DE-SC0012152

310 and DE-SC00-12548) as well as the NSF (AGS-1734164). Stephan Rasp was funded by the

311 German Research Foundation (DFG) Transregional Collaborative Research Center SFB/ TRR

312165 "Waves to Weather'. Computational resources for our SPCAM3 simulations were provided

313 through the NSF Extreme Science and Engineering Discovery Environment (XSEDE) under

314 allocation TG-ATM120034. 
Figures

\begin{tabular}{|c|c|c|c|}
\hline Input variables & Dimensionality & Output variables & Dimensionality \\
beginning of time step & $\begin{array}{c}\text { Time, lat, lon, } \\
\text { level }\end{array}$ & $\begin{array}{c}\text { Convective and turbulent } \\
\text { temperature tendency }\end{array}$ & $\begin{array}{c}\text { Time, lat, lon, } \\
\text { level }\end{array}$ \\
\hline $\begin{array}{c}\text { Humidity at beginning of } \\
\text { time step }\end{array}$ & $\begin{array}{c}\text { Time, lat, lon } \\
\text { level }\end{array}$ & $\begin{array}{c}\text { Convective and turbulent } \\
\text { humidity tendency }\end{array}$ & $\begin{array}{c}\text { Time, lat, lon, } \\
\text { level }\end{array}$ \\
\hline Surface pressure & Time, lat, lon & Longwave heating tendency & $\begin{array}{c}\text { Time, lat, lon, } \\
\text { level }\end{array}$ \\
\hline Sensible heat flux & Time, lat, lon & Shortwave heating tendency & $\begin{array}{c}\text { Time, lat, lon, } \\
\text { level }\end{array}$ \\
\hline Latent heat flux & Time, lat, lon & & \\
\hline $\begin{array}{c}\text { Temperature tendency } \\
\text { from dynamics }\end{array}$ & $\begin{array}{c}\text { Time, lat, lon } \\
\text { level }\end{array}$ & & \\
\hline $\begin{array}{c}\text { Humidity tendency from } \\
\text { dynamics }\end{array}$ & $\begin{array}{c}\text { Time, lat, lon } \\
\text { level }\end{array}$ & & \\
\hline $\begin{array}{c}\text { Incoming solar radiation } \\
\text { Size of stacked array }\end{array}$ & $\begin{array}{l}\text { Time, lat, lon } \\
124\end{array}$ & & 120 \\
\hline
\end{tabular}

317 Table 1: List of input and output variables used for the neural network. 

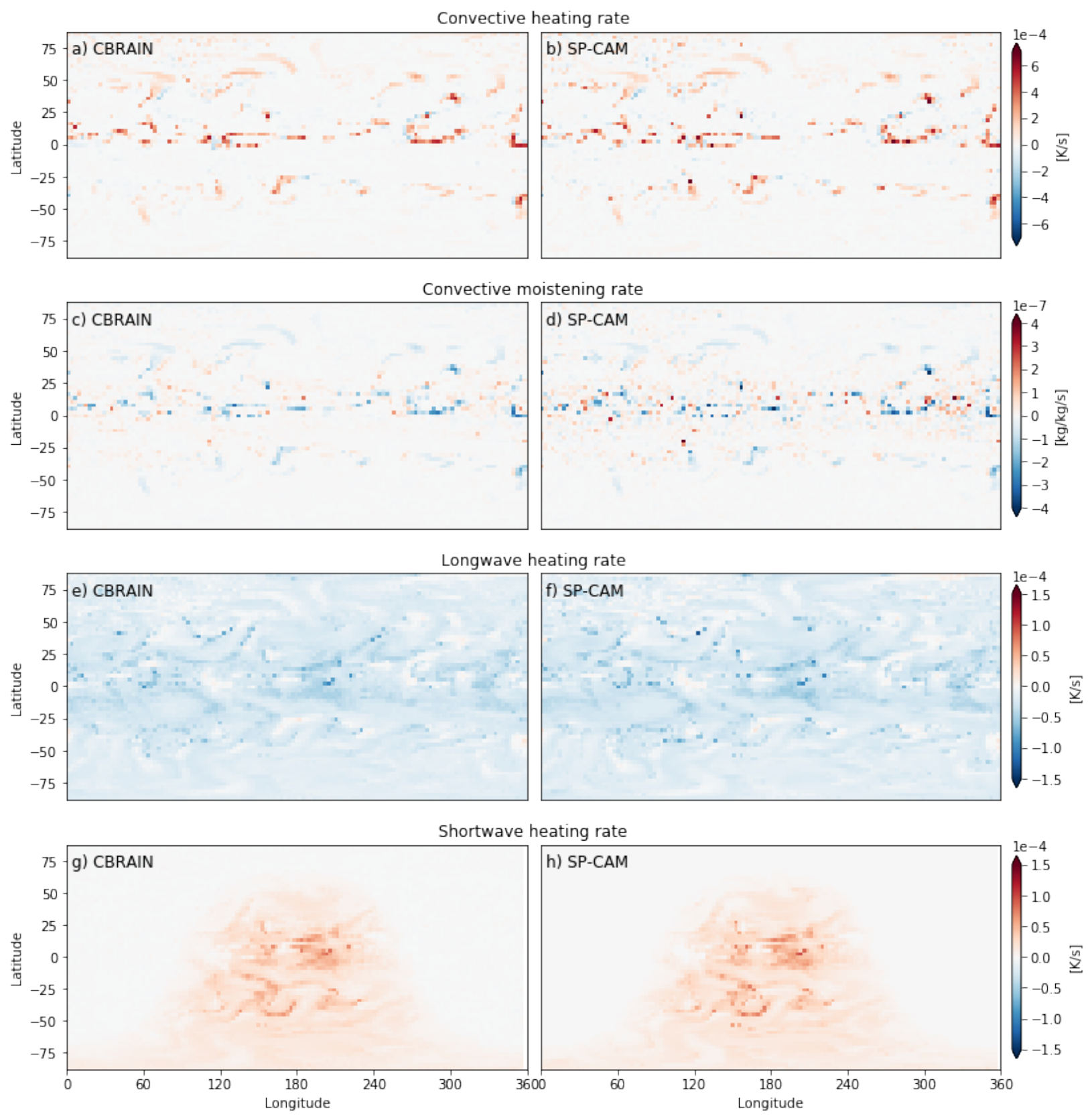

320 Figure 1: Latitude-longitude snapshot of neural network predictions and the corresponding SP-

321 CAM truth at model level 20 (roughly $700 \mathrm{hPa}$ ) for one time step in the validation set. 

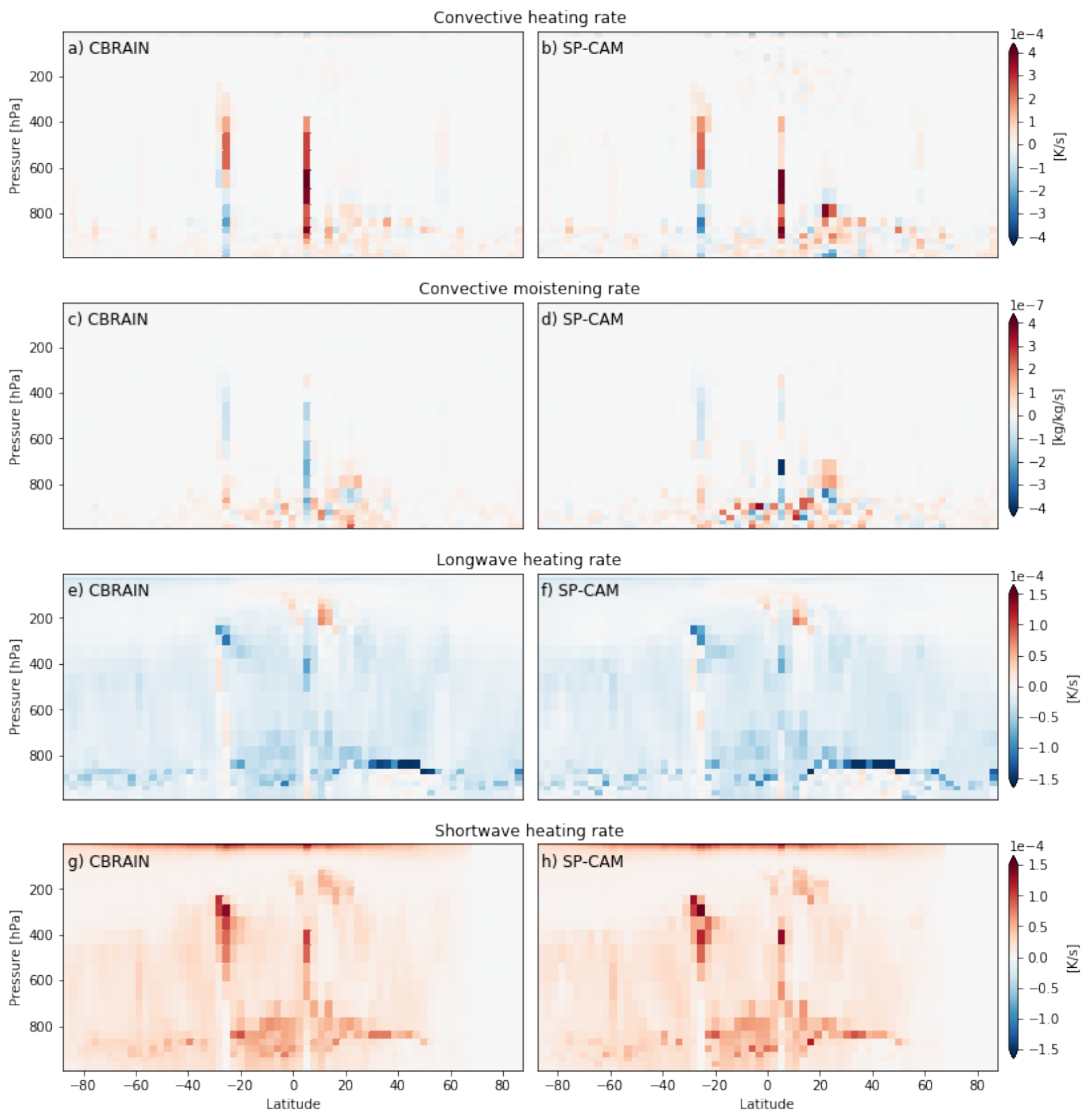

325 Figure 2: Pressure-latitude snapshot at $180^{\circ}$ longitude corresponding to Figure 3. 


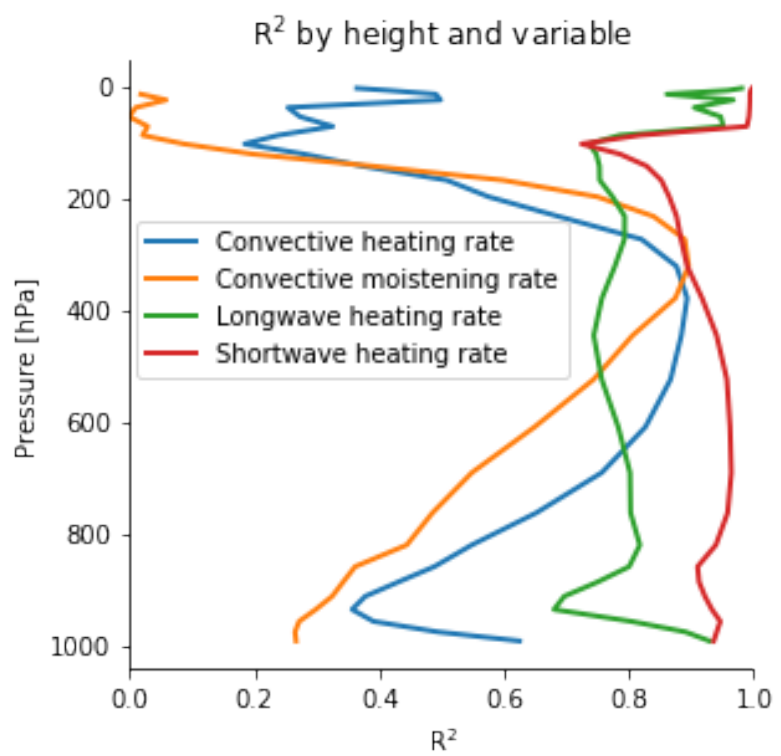

Figure 3: $\mathrm{R}^{2}$ computed for each model pressure level and variable as described in the text.
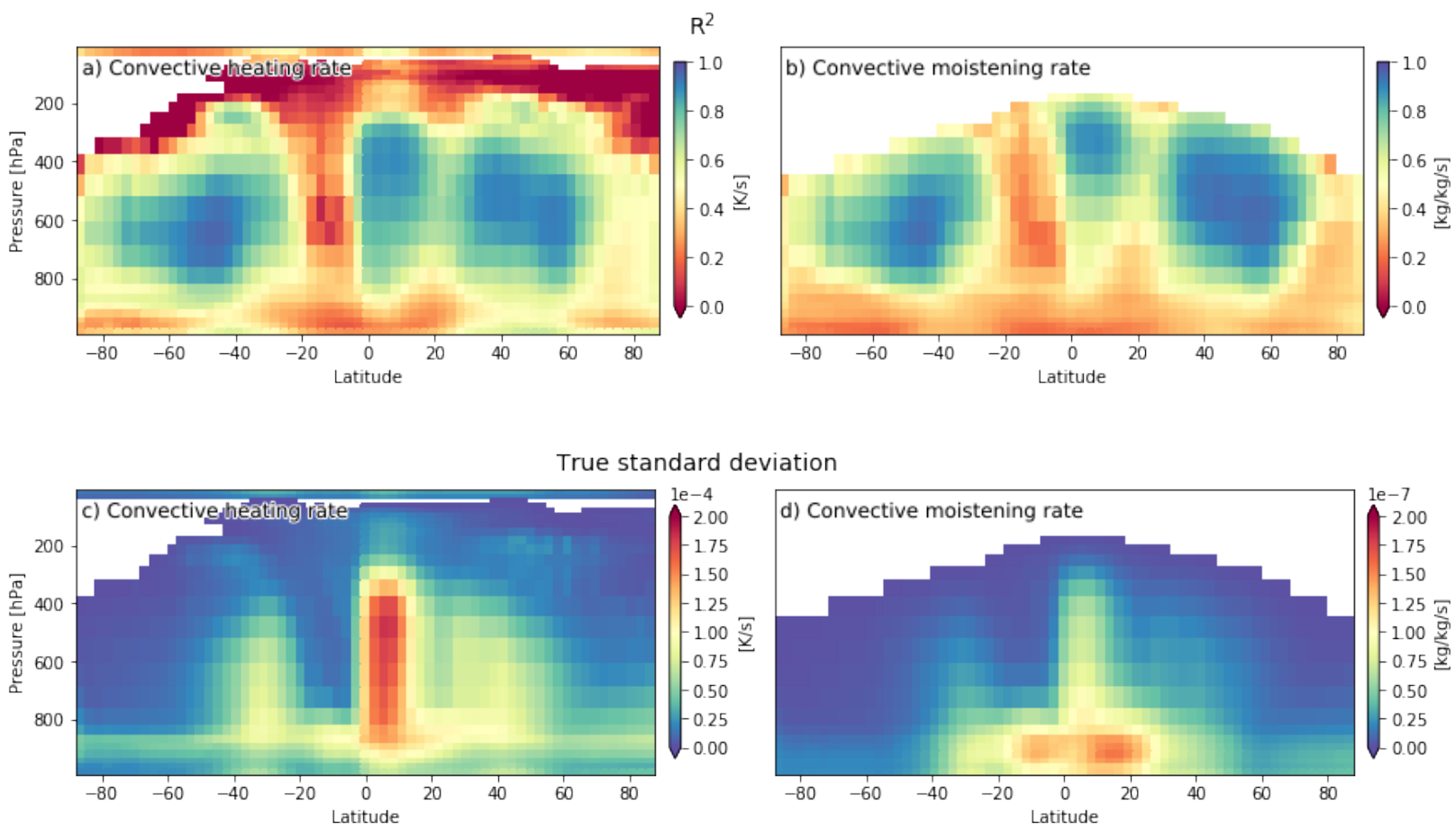

Figure 4: Pressure-latitude maps of (top row) $\mathrm{R}^{2}$ and (bottom row) true standard deviation

332 averaged over time and longitude. Regions where the variance was less than $0.05 \%$ of the global

333 variance were masked out. 


\begin{tabular}{|l|l|l|l|l|}
\hline $\begin{array}{l}\text { Approximate } \\
\text { number of } \\
\text { parameters }\end{array}$ & $30 \mathrm{k}$ & $125 \mathrm{k}$ & $500 \mathrm{k}$ & $2 \mathrm{M}$ \\
\hline Shallow & 128 & 512 & 2048 & 8192 \\
\hline Medium & $90 \times 2$ & $256 \times 2$ & $600 \times 2$ & $1300 \times 2$ \\
\hline Deep & $50 \times 8$ & $115 \times 8$ & $256 \times 8$ & $512 \times 8$ \\
\hline
\end{tabular}

336 nodes. The numbers in the table represent the nodes in the fully connected hidden layers. Note

337 that powers of two are commonly chosen to speed up computations on the GPU.

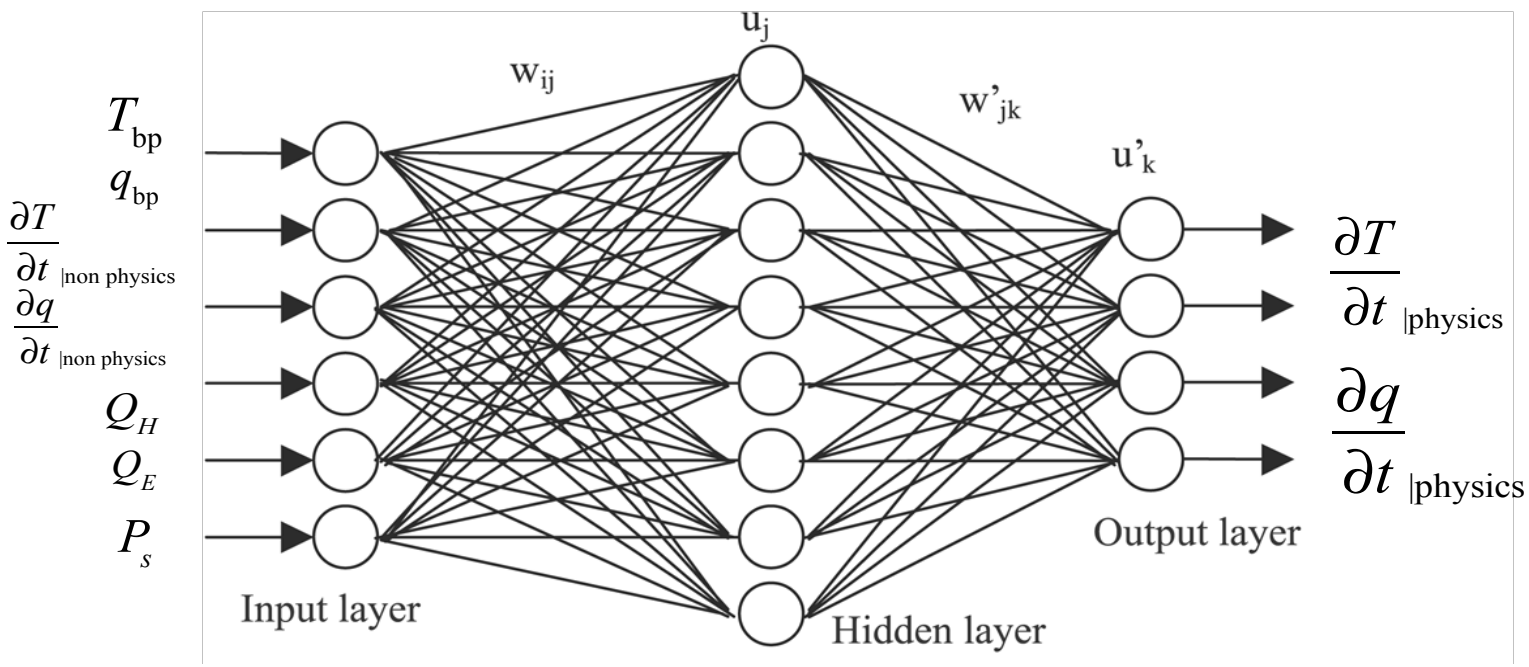



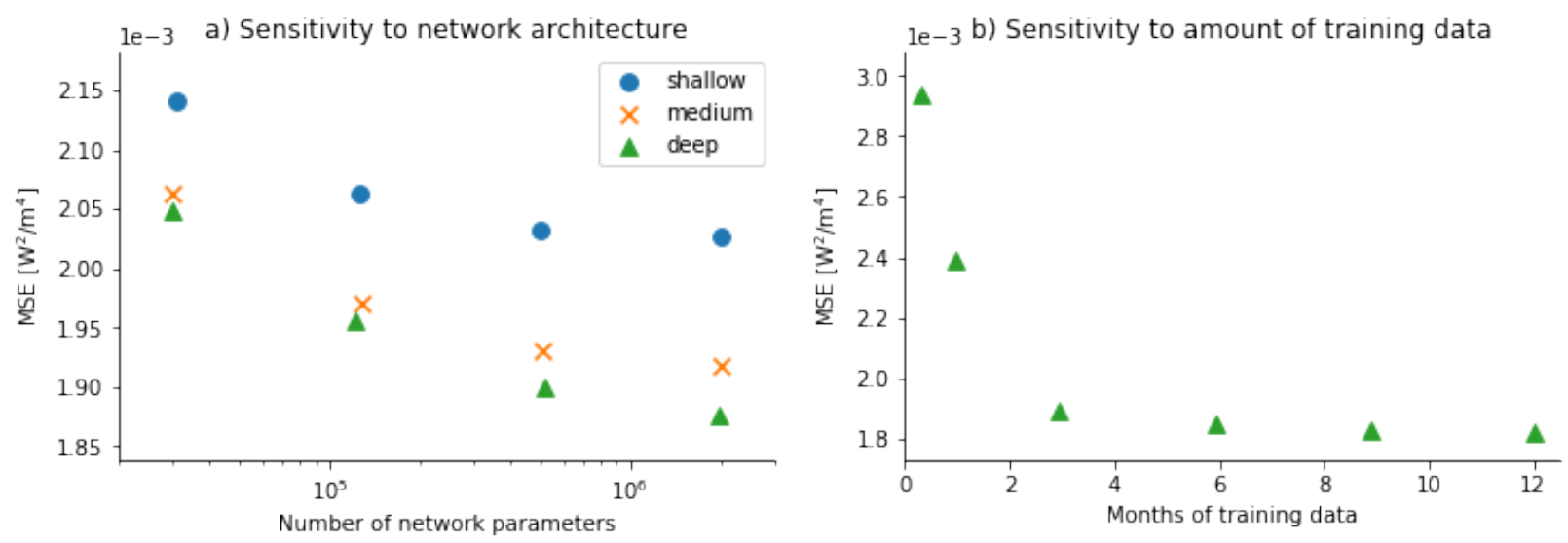

Figure S 2: Sensitivity tests to (a) network architecture and (b) amount of training data. The

345

346

347

score is the mean squared error averaged over time, space and variables in energy units computed from the validation set.

Andersen, J. A., and Z. Kuang (2012), Moist static energy budget of MJO-like disturbances in the atmosphere of a zonally symmetric aquaplanet, J of Climate, 25(8), 2782-2804, doi:10.1175/JCLI-D-11-00168.1.

Arnold, N. P., and D. A. Randall (2015), Global-scale convective aggregation: Implications for the Madden-Julian Oscillation, J. Adv. Model. Earth Syst., 7(4), 1499-1518, doi:10.1002/2015MS000498.

Arnold, N. P., M. Branson, M. A. Burt, D. S. Abbot, Z. Kuang, D. A. Randall, and E. Tziperman (2014), Effects of explicit atmospheric convection at high CO2, PNAS, 111(30), 1094310948, doi:10.1073/pnas. 1407175111.

Arnold, N. P., M. Branson, Z. Kuang, and D. A. Randall (2015), MJO intensification with warming in the superparameterized CESM, J Climate, doi:10.1175/JCLI-D-14-00494.1.

Benedict, J. J., and M. S. Pritchard (2015), Sensitivity of MJO propagation to a robust positive Indian Ocean dipole event in the superparameterized CAM, Journal of Advances in Modeling Earth Systems, doi:10.1002/2015MS000530/pdf.

Bony, S. et al. (2015), Clouds, circulation and climate sensitivity, Nat Geosci, 8(4), 261-268, doi:10.1038/ngeo2398.

Bretherton, C. S., and M. F. Khairoutdinov (2015), Convective self-aggregation feedbacks in near-global cloud-resolving simulations of an aquaplanet, J. Adv. Model. Earth Syst., 7(4), 
1765-1787, doi:10.1002/2015MS000499.

Bush, S. J., A. G. Turner, S. J. Woolnough, G. M. Martin, and N. P. Klingaman (2015), The effect of increased convective entrainment on Asian monsoon biases in the MetUM general circulation model, Q J Roy Meteor Soc, 141(686), 311-326, doi:10.1002/qj.2371.

Cheng, A., and K.-M. Xu (2014), An Explicit Representation of Vertical Momentum Transport in a Multiscale Modeling Framework through its 2D Cloud-Resolving Model Component, $J$ Geophys Res-Atmos, n/a-n/a, doi:10.1002/2013JD021078.

Chiang, J., and A. H. Sobel (2002), Tropical tropospheric temperature variations caused by ENSO and their influence on the remote tropical climate, J Climate, doi:10.1175/15200442(2002)015.

Cohen, C. (2000), A Quantitative Investigation of Entrainment and Detrainment in Numerically Simulated Cumulonimbus Clouds, J Atmos Sci, 57(10), 1657-1674.

Coppin, D., and S. Bony (2015), Physical mechanisms controlling the initiation of convective self-aggregation in a General Circulation Model, J. Adv. Model. Earth Syst., 7(4), 20602078, doi:10.1002/2015MS000571.

Couvreux, F. et al. (2015), Representation of daytime moist convection over the semi-arid Tropics by parametrizations used in climate and meteorological models, $Q J$ Roy Meteor Soc, 141(691), 2220-2236, doi:10.1002/qj.2517.

D'Andrea, F., P. Gentine, A. K. Betts, and B. R. Lintner (2014), Triggering Deep Convection with a Probabilistic Plume Model, Journal of the atmospheric ..., 71(11), 3881-3901, doi:10.1175/JAS-D-13-0340.1.

Dahl, G. E., Dong Yu, Li Deng, and A. Acero (2011), Context-Dependent Pre-Trained Deep Neural Networks for Large-Vocabulary Speech Recognition, IEEE Trans. Audio Speech Lang. Process., 20(1), 30-42, doi:10.1109/TASL.2011.2134090.

Daleu, C. L. et al. (2015), Intercomparison of methods of coupling between convection and large-scale circulation: 1. Comparison over uniform surface conditions, J. Adv. Model. Earth Syst., 7(4), 1576-1601, doi:10.1002/2015MS000468.

Daleu, C. L. et al. (2016), Intercomparison of methods of coupling between convection and large-scale circulation: 2. Comparison over nonuniform surface conditions, J. Adv. Model. Earth Syst., n/a-n/a, doi:10.1002/2015MS000570.

Dawe, J. T., and P. H. Austin (2013), Direct entrainment and detrainment rate distributions of individual shallow cumulus clouds in an LES, Atmos. Chem. Phys, 13(15), 7795-7811, doi:10.5194/acp-13-7795-2013.

De Rooy, W. C., and A. P. Siebesma (2010), Analytical expressions for entrainment and detrainment in cumulus convection, Q J Roy Meteor Soc, 136(650), 1216-1227, doi:10.1002/qj.640. 
De Rooy, W. C., P. Bechtold, K. Froehlich, C. Hohenegger, H. Jonker, D. Mironov, A. P. Siebesma, J. Teixeira, and J.-I. Yano (2013), Entrainment and detrainment in cumulus convection: an overview, Q J Roy Meteor Soc, 139(670), 1-19, doi:10.1002/qj.1959.

Del Genio, A. D., and J. Wu (2010), The Role of Entrainment in the Diurnal Cycle of Continental Convection, J Climate, 23(10), 2722-2738, doi:10.1175/2009JCLI3340.1.

Derbyshire, S., I. Beau, P. Bechtold, J. Grandpeix, J. Piriou, J. Redelsperger, and P. M. M. Soares (2004), Sensitivity of moist convection to environmental humidity, $Q J$ Roy Meteor Soc, 130(604), 3055-3079, doi:10.1256/qj.03.130.

Dorrestijn, J., D. T. Crommelin, A. P. Siebesma, H. J. J. Jonker, and C. Jakob (2015), Stochastic Parameterization of Convective Area Fractions with a Multicloud Model Inferred from Observational Data, Journal of Atmospheric Sciences, 72(2), 854-869, doi:10.1175/JAS-D14-0110.1.

Feng, Z., S. Hagos, A. K. Rowe, C. D. Burleyson, M. N. Martini, and S. P. de Szoeke (2015), Mechanisms of convective cloud organization by cold pools over tropical warm ocean during the AMIE/DYNAMO field campaign, J. Adv. Model. Earth Syst., 7(2), 357-381, doi:10.1002/2014MS000384.

Gentine, P., A. Garelli, S. B. Park, and J. Nie (2016), Role of surface heat fluxes underneath cold pools, Geo Res Letters, 43(2), 874-883, doi:10.1002/2015GL067262.

Goswami, B. B., P. Mukhopadhyay, M. Khairoutdinov, and B. N. Goswami (2013), Simulation of Indian summer monsoon intraseasonal oscillations in a superparameterized coupled climate model: need to improve the embedded cloud resolving model, Climate dynamics, 41(5-6), 1497-1507, doi:10.1007/s00382-012-1563-1.

Guichard, F. et al. (2004), Modelling the diurnal cycle of deep precipitating convection over land with cloud-resolving models and single-column models, QJ Roy Meteor Soc, 130(604), 3139-3172, doi:10.1256/qj.03.145.

Hinton, G. et al. (2012), Deep Neural Networks for Acoustic Modeling in Speech Recognition: The Shared Views of Four Research Groups, IEEE Signal Process. Mag., 29(6), 82-97, doi:10.1109/MSP.2012.2205597.

Hohenegger, C., and B. Stevens (2016), Coupled radiative convective equilibrium simulations with explicit and parameterized convection, J. Adv. Model. Earth Syst., 8(3), 1468-1482, doi:10.1002/2016MS000666.

Hohenegger, C., L. Schlemmer, and L. Silvers (2015), Coupling of convection and circulation at various resolutions, Tellus Series A-Dynamic Meteorology And Oceanography, 67(0), doi:10.3402/tellusa.v67.26678.

Hohenegger, C., P. Brockhaus, C. S. Bretherton, and C. Schaer (2009), The Soil MoisturePrecipitation Feedback in Simulations with Explicit and Parameterized Convection, $J$ Climate, 22(19), 5003-5020, doi:10.1175/2009JCLI2604.1. 
440

Houze, R. A. (2004), Mesoscale convective systems, Reviews of Geophysics, 42(4), RG4003, doi:10.1029/2004RG000150.

Jeevanjee, N., and D. M. Romps (2013), Convective self-aggregation, cold pools, and domain size, Geophys Res Lett, 40(5), 994-998, doi:10.1002/grl.50204.

Jimenez, C., C. Prigent, and F. Aires (2009), Toward an estimation of global land surface heat fluxes from multisatellite observations, J Geophys Res-Atmos, 114(D6), D06305-, doi:10.1029/2008JD011392.

Karabatak, M., and M. C. Ince (2009), An expert system for detection of breast cancer based on association rules and neural network, Expert Systems With Applications, 36(P2), 3465-3469, doi:10.1016/j.eswa.2008.02.064.

Khairoutdinov, M. F., S. K. Krueger, C.-H. Moeng, P. A. Bogenschutz, and D. A. Randall (2009), Large-eddy simulation of maritime deep tropical convection, J. Adv. Model. Earth Syst., 2, 15, doi:10.3894/JAMES.2009.1.15.S1.

Khairoutdinov, M., and D. Randall (2006), High-resolution simulation of shallow-to-deep convection transition over land, J Atmos Sci, 63(12), 3421-3436.

Khairoutdinov, M., D. Randall, and C. DeMott (2005), Simulations of the Atmospheric General Circulation Using a Cloud-Resolving Model as a Superparameterization of Physical Processes, Journal of Atmospheric Sciences, 62(7), 2136-2154, doi:10.1175/JAS3453.1.

Khan, J., J. S. Wei, M. Ringner, L. H. Saal, and M. Ladanyi (2001), Classification and diagnostic prediction of cancers using gene expression profiling and artificial neural networks, Nature.

Khouider, B., A. Majda, and M. Katsoulakis (2003), Coarse-grained stochastic models for tropical convection and climate, P Natl Acad Sci Usa, 100(21), 11941-11946, doi:10.1073/pnas. 1634951100 .

Khouider, B., and A. Majda (2006), A simple multicloud parameterization for convectively coupled tropical waves. Part I: Linear analysis, J Atmos Sci, 63(4), 1308-1323.

Khouider, B., J. Biello, and A. J. Majda (2010), A stochastic multicloud model for tropical convection, Commun Math Sci, 8(1), 187-216.

Kim, D., A. H. Sobel, A. D. Del Genio, Y. Chen, S. J. Camargo, M.-S. Yao, M. Kelley, and L. Nazarenko (2012), The Tropical Subseasonal Variability Simulated in the NASA GISS General Circulation Model, J Climate, 25(13), 4641-4659, doi:10.1175/JCLI-D-11-00447.1.

Kingma, D. P., and J. Ba (2014), Adam: A Method for Stochastic Optimization, arXiv, cs.LG.

Kolassa, J., F. Aires, J. Polcher, C. Prigent, C. Jimenez, and J. M. Pereira (2013), Soil moisture retrieval from multi-instrument observations: Information content analysis and retrieval methodology, J Geophys Res-Atmos, 118(10), 4847-4859, doi:10.1029/2012JD018150. 
Kolassa, J., P. Gentine, C. Prigent, and F. Aires (2016), Soil moisture retrieval from AMSR-E and ASCAT microwave observation synergy. Part 1: Satellite data analysis, Remote Sensing of Environment, 173(C), 1-14, doi:10.1016/j.rse.2015.11.011.

Kolassa, J., P. Gentine, C. Prigent, F. Aires, and S. H. Alemohammad (2017a), Soil moisture retrieval from AMSR-E and ASCAT microwave observation synergy. Part 2: Product evaluation, Remote Sensing of Environment, 195, 202-217, doi:10.1016/j.rse.2017.04.020.

Kolassa, J., R. H. Reichle, and C. S. Draper (2017b), Merging active and passive microwave observations in soil moisture data€assimilation, Remote Sensing of Environment, 191(C), 117-130, doi:10.1016/j.rse.2017.01.015.

Kooperman, G. J., M. S. Pritchard, and R. C. J. Somerville (2013), Robustness and sensitivities of central US summer convection in the super-parameterized CAM: Multi-model intercomparison with a new regional EOF index, Geophys Res Lett, 40(12), 3287-3291, doi:10.1002/grl.50597.

Kooperman, G. J., M. S. Pritchard, and R. C. J. Somerville (2014), The response of US summer rainfall to quadrupled $\mathrm{CO} 2$ climate change in conventional and superparameterized versions of the NCAR Community Atmosphere Model, J. Adv. Model. Earth Syst., n/a-n/a, doi:10.1002/2014MS000306.

Kooperman, G. J., M. S. Pritchard, M. A. Burt, M. D. Branson, and D. A. Randall (2016a), Impacts of cloud superparameterization on projected daily rainfall intensity climate changes in multiple versions of the Community Earth System Model, J. Adv. Model. Earth Syst., 173, doi:10.1002/2016MS000715.

Kooperman, G. J., M. S. Pritchard, M. A. Burt, M. D. Branson, and D. A. Randall (2016b), Robust effects of cloud superparameterization on simulated daily rainfall intensity statistics across multiple versions of the Community Earth System Model, J. Adv. Model. Earth Syst., n/a-n/a, doi:10.1002/2015MS000574.

LeCun, Y., Y. Bengio, and G. Hinton (2015), Deep learning, Nature, 521(7553), 436-444, doi:10.1038/nature14539.

Mapes, B., and R. Neale (2011), Parameterizing Convective Organization to Escape the Entrainment Dilemma, J. Adv. Model. Earth Syst., 3(6), M06004, doi:10.1029/2011MS000042.

Medeiros, B., B. Stevens, and S. Bony (2014), Using aquaplanets to understand the robust responses of comprehensive climate models to forcing, Climate dynamics, 44(7-8), 19571977, doi:10.1007/s00382-014-2138-0.

Miao, C., H. Ashouri, K.-L. Hsu, S. Sorooshian, and Q. Duan (2015), Evaluation of the PERSIANN-CDR Daily Rainfall Estimates in Capturing the Behavior of Extreme Precipitation Events over China, J Hydrometeorol, 16(3), 1387-1396, doi:10.1175/JHM-D14-0174.1. 
Moazami, S., S. Golian, M. R. Kavianpour, and Y. Hong (2013), Comparison of PERSIANN and V7 TRMM Multi- satellite Precipitation Analysis (TMPA) products with rain gauge data over Iran, International Journal of Remote Sensing, 34(22), 8156-8171, doi:10.1080/01431161.2013.833360.

Moncrieff, M. W., and C. Liu (2006), Representing convective organization in prediction models by a hybrid strategy, J Atmos Sci, 63(12), 3404-3420.

Muller, C., and S. Bony (2015), What favors convective aggregation and why? Geophys Res Lett, 42(13), 5626-5634, doi:10.1002/2015GL064260.

Naumann, A. K., B. Stevens, C. Hohenegger, and J. P. Mellado (2017), A Conceptual Model of a Shallow Circulation Induced by Prescribed Low-Level Radiative Cooling, Journal of Atmospheric Sciences, 74(10), 3129-3144, doi:10.1175/JAS-D-17-0030.1.

Nie, J., D. A. Shaevitz, and A. H. Sobel (2016), Forcings and feedbacks on convection in the 2010 Pakistan flood: Modeling extreme precipitation with interactive large-scale ascent, $J$. Adv. Model. Earth Syst., 1-47, doi:10.1002/2016MS000663.

Oueslati, B., and G. Bellon (2015), The double ITCZ bias in CMIP5 models: interaction between SST, large-scale circulation and precipitation, Climate dynamics, doi:10.1007/s00382-0152468-6.

Parishani, H., M. S. Pritchard, C. S. Bretherton, M. C. Wyant, and M. Khairoutdinov (2017), Toward low cloud-permitting cloud superparameterization with explicit boundary layer turbulence, J. Adv. Model. Earth Syst., doi:10.1002/2017MS000968.

Peters, K., C. Jakob, L. Davies, B. Khouider, and A. J. Majda (2013), Stochastic behavior of tropical convection in observations and a multicloud model, J Atmos Sci, 130710123341008, doi:10.1175/JAS-D-13-031.1.

Popke, D., B. Stevens, and A. Voigt (2013), Climate and climate change in a radiativeconvective equilibrium version of ECHAM6, J. Adv. Model. Earth Syst., 5(1), 1-14, doi:10.1029/2012MS000191.

Pritchard, M. S., and R. C. J. Somerville (2009), Assessing the Diurnal Cycle of Precipitation in a Multi-Scale Climate Model, J. Adv. Model. Earth Syst., 1, doi:10.3894/JAMES.2009.1.12.

Pritchard, M. S., C. S. Bretherton, and C. A. Demott (2014), Restricting 32-128 km horizontal scales hardly affects the MJO in the Superparameterized Community Atmosphere Model v.3.0 but the number of cloud-resolving grid columns constrains vertical mixing, $J$. $A d v$. Model. Earth Syst., 6(3), 723-739, doi:10.1002/2014MS000340.

Pritchard, M. S., M. W. Moncrieff, and R. C. J. Somerville (2011), Orogenic Propagating Precipitation Systems over the United States in a Global Climate Model with Embedded Explicit Convection, Journal of Atmospheric Sciences, 68(8), 1821-1840, doi:10.1175/2011JAS3699.1. 
Rochetin, N., F. Couvreux, J.-Y. Grandpeix, and C. Rio (2014a), Deep Convection Triggering by Boundary Layer Thermals. Part I: LES Analysis and Stochastic Triggering Formulation, $J$ Atmos Sci, 71(2), 496-514, doi:10.1175/JAS-D-12-0336.1.

Rochetin, N., J.-Y. Grandpeix, C. Rio, and F. Couvreux (2014b), Deep Convection Triggering by Boundary Layer Thermals. Part II: Stochastic Triggering Parameterization for the LMDZ GCM, J Atmos Sci, 71(2), 515-538, doi:10.1175/JAS-D-12-0337.1.

Romps, D. M. (2010), A Direct Measure of Entrainment, J Atmos Sci, 67(6), 1908-1927, doi:10.1175/2010JAS3371.1.

Romps, D. M. (2016), The Stochastic Parcel Model: A deterministic parameterization of stochastically entraining convection, J. Adv. Model. Earth Syst., n/a-n/a, doi:10.1002/2015MS000537.

Satoh, M., T. Matsuno, H. Tomita, H. Miura, T. Nasuno, and S. Iga (2008), Nonhydrostatic icosahedral atmospheric model (NICAM) for global cloud resolving simulations, Journal of Computational Physics, 227(7), 3486-3514, doi:10.1016/j.jcp.2007.02.006.

Schmidhuber, J. (2015), Deep learning in neural networks: An overview, Neural Networks, 61, 85-117, doi:10.1016/j.neunet.2014.09.003.

Schneider, T., J. Teixeira, C. S. Bretherton, F. Brient, K. G. Pressel, C. Schär, and A. P. Siebesma (2017), Climate goals and computing the future of clouds, Nature Climate Change, 7(1), 3-5, doi:10.1038/nclimate3190.

Sherwood, S. C., and D. Hernández-Deckers (2013), Slippery thermals and the cumulus entrainment paradox*, J Atmo Sci, 70(8), 2426-2442.

Sherwood, S. C., S. Bony, and J. L. Dufresne (2014), Spread in model climate sensitivity traced to atmospheric convective mixing, Nature, 505(7481), 37-42, doi:10.1038/nature12829.

Silver, D. et al. (2016), Mastering the game of Go with deep neural networks and tree search, Nature, 529(7585), 484-489, doi:10.1038/nature16961.

Singh, M. S., and P. A. O'Gorman (2013), Influence of entrainment on the thermal stratification in simulations of radiative-convective equilibrium, Geophys Res Lett, 40(16), 4398-4403, doi:10.1002/grl.50796.

Sobel, A. H., J. Nilsson, and L. M. Polvani (2001), The weak temperature gradient approximation and balanced tropical moisture waves, Journal of Atmospheric Sciences, 58(23), 3650-3665.

Stevens, B., and S. Bony (2013), What Are Climate Models Missing? Science, 340(6136), 10531054, doi:10.1126/science.1237554.

Subramanian, A. C., and T. N. Palmer (2017), Ensemble superparameterization versus stochastic parameterization: A comparison of model uncertainty representation in tropical weather 
Confidential manuscript submitted to Geophysical Research Letters

prediction, J. Adv. Model. Earth Syst., doi:10.1002/2016MS000857.

Sun, J., and M. S. Pritchard (2016), Effects of explicit convection on global land-atmosphere coupling in the superparameterized CAM, J. Adv. Model. Earth Syst., 8(3), 1248-1269, doi:10.1002/2016MS000689.

Tan, J., C. Jakob, W. B. Rossow, and G. Tselioudis (2015), Increases in tropical rainfall driven by changes in frequency of organized deep convection, Nature, 519(7544), 451-+, doi:10.1038/nature14339.

Tao, Y., X. Gao, K. Hsu, S. Sorooshian, and A. Ihler (2016), A Deep Neural Network Modeling Framework to Reduce Bias in Satellite Precipitation Products, J Hydrometeorol, 17(3), 931945, doi:10.1175/JHM-D-15-0075.1.

Taylor, C. M., C. E. Birch, D. J. Parker, N. Dixon, F. Guichard, G. Nikulin, and G. M. S. Lister (2013), Modelling soil moisture - precipitation feedbacks in the Sahel: importance of spatial scale versus convective parameterization, Geophys Res Lett, 40(23), 6213-6218, doi:10.1002/2013GL058511.

Taylor, C. M., P. P. Harris, and D. J. Parker (2009), Impact of soil moisture on the development of a Sahelian mesoscale convective system: a case-study from the AMMA Special Observing Period, Q J Roy Meteor Soc, 136(S1), 456-470, doi:10.1002/qj.465.

Tian, Y., and Z. Kuang (2016), Dependence of entrainment in shallow cumulus convection on vertical velocity and distance to cloud edge, Geophys Res Lett, 43(8), 4056-4065, doi:10.1002/2016GL069005.

Tomassini, L., A. Voigt, and B. Stevens (2014), On the connection between tropical circulation, convective mixing, and climate sensitivity, $Q J$ Roy Meteor Soc, n/a-n/a, doi:10.1002/qj.2450.

Tulich, S. N. (2015), A strategy for representing the effects of convective momentum transport in multiscale models: Evaluation using a new superparameterized version of the Weather Research and Forecast model (SP-WRF), J. Adv. Model. Earth Syst., 7(2), 938-962, doi:10.1002/2014MS000417.

Wang, S., and A. H. Sobel (2011), Response of convection to relative sea surface temperature: Cloud-resolving simulations in two and three dimensions, J Geophys Res, 116(D11), D11119, doi:10.1029/2010JD015347.

Wing, A. A., and K. A. Emanuel (2014), Physical mechanisms controlling self-aggregation of convection in idealized numerical modeling simulations, J. Adv. Model. Earth Syst., 6(1), 59-74, doi:10.1002/2013MS000269.

Yeo, K., and D. M. Romps (2013), Measurement of Convective Entrainment Using Lagrangian Particles, J Atmos Sci, 70(1), 266-277, doi:10.1175/JAS-D-12-0144.1. 
617 Yu, S., and M. S. Pritchard (2015), The effect of large-scale model time step and multiscale 618 coupling frequency on cloud climatology, vertical structure, and rainfall extremes in a superparameterized GCM, J. Adv. Model. Earth Syst., 7(4), 1977-1996,

621 Zhou, Z. H., Y. Jiang, Y. B. Yang, and S. F. Chen (2002), Lung cancer cell identification based 622 on artificial neural network ensembles, Artificial Intelligence in Medicine, 24(1), 25-36. 